\title{
Neural Correlates of Dispositional Mindfulness During Affect Labeling
}

\author{
J. David Creswell, PhD, Baldwin M. Way, PhD, Naomi I. Eisengerger, PhD, and Matthew D. Lieberman, PhD
}

\begin{abstract}
Objective: Mindfulness is a process whereby one is aware and receptive to present moment experiences. Although mindfulnessenhancing interventions reduce pathological mental and physical health symptoms across a wide variety of conditions and diseases, the mechanisms underlying these effects remain unknown. Converging evidence from the mindfulness and neuroscience literature suggests that labeling affect may be one mechanism for these effects. Methods: Participants $(n=27)$ indicated trait levels of mindfulness and then completed an affect labeling task while undergoing functional magnetic resonance imaging. The labeling task consisted of matching facial expressions to appropriate affect words (affect labeling) or to gender-appropriate names (gender labeling control task). Results: After controlling for multiple individual difference measures, dispositional mindfulness was associated with greater widespread prefrontal cortical activation, and reduced bilateral amygdala activity during affect labeling, compared with the gender labeling control task. Further, strong negative associations were found between areas of prefrontal cortex and right amygdala responses in participants high in mindfulness but not in participants low in mindfulness. Conclusions: The present findings with a dispositional measure of mindfulness suggest one potential neurocognitive mechanism for understanding how mindfulness meditation interventions reduce negative affect and improve health outcomes, showing that mindfulness is associated with enhanced prefrontal cortical regulation of affect through labeling of negative affective stimuli. Key words: fMRI, mindfulness, emotion regulation, neuroscience, meditation, negative affect.
\end{abstract}

fMRI = functional magnetic resonance imaging; PFC $=$ prefrontal cortex; VLPFC = ventrolateral prefrontal cortex; VMPFC $=$ ventromedial prefrontal cortex; MPFC = medial prefrontal cortex; DLPFC = dorsolateral prefrontal cortex.

The skillful use of labeling during satipatthana [mindful] contemplation can help strengthen clear recognition and understanding. At the same time, labeling introduces a healthy degree of inner detachment, since the act of apostrophizing one's moods and emotions diminishes one's identification with them.

Analayo, from Satipatthana

\section{INTRODUCTION}

$\mathrm{M}$ indfulness is a process whereby one is aware and receptive to present moment experiences (1) and has been suggested to be at the "heart" of Buddhist meditation practices (2). Over the last two decades, mindfulness has received a great deal of attention in the behavioral medicine and psychiatric literatures, resulting in the development of multiple mindfulness meditation treatments designed to treat a variety of conditions and diseases (3). Studies consistently show that mindful traits (1) and mindfulness meditation practices (3) reduce negative affect, stress, mood disturbance, and diseasespecific health symptoms across many patient populations. Despite these benefits, it is not clear how mindfulness produces these effects.

Labeling aspects of experience is a central feature of historical and contemporary accounts of mindfulness, and may represent one mechanism for the salutary effects of mindfulness practice. In an interpretation of the Buddha's original

From the Department of Psychology (J.D.C., B.M.W., M.D.L.) and Cousins Center for Psychoneuroimmunology (N.I.E.), University of California, Los Angeles, Los Angeles, California.

Address correspondence and reprint requests to J. David Creswell, Department of Psychology, University of California, 405 Hilgard Ave, Los Angeles, CA 90095-1563. E-mail: creswell@ucla.edu or davidcreswell@hotmail.com

Received for publication September 18, 2006; revision received April 2, 2007.

This research was supported by a National Institute of Mental Health (NIMH) predoctoral NRSA research fellowship (J.D.C.) and NIMH postdoctoral research fellowships MH15750 (B.M.W.; part of the UCLA Health Psychology Program) and T32MH-019925 (N.I.E.), as well as by NIMH grants R21MH66709 and R21MH071521 (M.D.L.).

DOI: 10.1097/PSY.0b013e3180f6171f discourse on mindfulness, Analayo quoted above described how labeling one's emotions through words promotes more effective recognition of, detachment from, and regulation of affective experiences (4). Similarly, contemporary mindfulness meditation teachers and interventions commonly prescribe labeling practices during mindfulness meditation (5-7). For example, a cognitive behavioral mindfulness meditation intervention (Mindfulness-Based Cognitive Therapy) encourages participants to use words or phrases during meditation to label emotional states (e.g., "here is anger") (6). Finally, labeling is an important facet of mindfulness in psychometric work with self-report mindfulness measures. The labeling subscale in one self-report mindfulness measure was associated with higher life satisfaction and improved emotion regulation (sample item: "I'm good at finding the words to describe my feelings") (8).

Accounts of affect labeling in the mindfulness tradition are in accord with a variety of therapeutic treatments (9) and recent findings in the neuroscience literature. For example, using functional neuroimaging, we and others have shown that verbally labeling affective stimuli activates right ventrolateral prefrontal cortex (VLPFC) and attenuates responses in the amygdala (a region commonly associated with negative affective states) $(9-11)$. Theoretical accounts of this functional neural network suggest that this pattern of activation is driven by top-down prefrontal cortex (PFC) inhibition of limbic responses (12), with some evidence suggesting that this inhibitory pathway occurs through connections in the medial prefrontal cortex (MPFC) $(9,13)$. This process of verbally labeling affective stimuli may disrupt or inhibit automatic affective responses, reducing their intensity and duration (9). Previous studies have shown that comparable neural responses during emotion regulation exercises are associated with reduced subjective reports of anxiety and negative affect (14-16).

In the present investigation, we bring together the converging lines of evidence in the mindfulness and neuroscience literatures, and test whether mindfulness is associated with enhanced neural regulation of affect during an affect labeling versus gender labeling control task. Specifically, we tested the hypothesis that dispositional mindfulness would be associated with greater activation in areas of the PFC (MPFC, right 
VLPFC), and would be associated with a concomitant deactivation of the amygdala during affect labeling.

\section{METHOD \\ Participants}

Twenty-seven UCLA undergraduates $(n=16$ females) participated in the study for course credit. Participants identified themselves as Asian (39\%), Caucasian (29\%), Latino (18\%), African American (7\%), or "other" (7\%). Prospective participants were excluded if they had serious mental/physical health problems, received mental health treatment, used psychotropic medication, or were pregnant/breast feeding. Participants also met the following functional magnetic resonance imaging (fMRI)-related inclusion criteria: a) were right handed; b) were not claustrophobic; and c) had no metal in their bodies (dental fillings were allowed). All procedures were approved by the UCLA Institutional Review Board. The study was conducted from June 2004 to January 2005.

\section{Procedure}

Participants completed a battery of individual difference measures, including a measure of dispositional mindfulness called the Mindful Attention Awareness Scale (MAAS) (1). This measure assesses one's general tendency to be open and receptive to present moment experiences across cognitive, emotional, physical, interpersonal, and general life domains. Next, the participants completed the labeling tasks while undergoing fMRI. In a blocked design, the participants viewed target faces displaying emotionally expressive faces and they were asked to perform two tasks (Figure 1). During the affect labeling task, participants chose the affect label from a pair of words shown at the bottom of the screen ("angry," "scared") that matched the target face. During the gender labeling task, the participants chose the gender-appropriate name from a pair of names shown at the bottom of the screen ("Samuel," "Helen") that matched the target face. This gender labeling task is a comparison condition that controls for the general cognitive processing demands required for the affect labeling task. Half of the target faces were male and half were female. The stimulus faces were counterbalanced across subjects for the affect and gender labeling tasks.

Task blocks began with a 3 -second instruction cue indicating the task type (affect label, gender label) followed by 10 randomized trials of the task, each 5 seconds in length, resulting in task blocks that were 50 seconds in length. Blocks were separated by a fixation crosshair, which remained on the screen for 10 seconds. The participants completed two affect labeling and two gender labeling blocks, administered in a randomized order. The participants responded via a button box as soon as they were sure of the correct answer. The stimuli remained on the screen for the entire 5 -second trial.

\section{Measures}

Participants completed the MAAS (1), a 15-item measure assessing trait levels of mindlessness (e.g., "I find it difficult to stay focused on what's happening in the present," all items were reverse scored; sample $\alpha=0.78$ ). Previous studies with undergraduates, community adults, and advanced meditation practitioners have shown that this measure has good psychometric properties and shows strong positive associations with multiple measures of

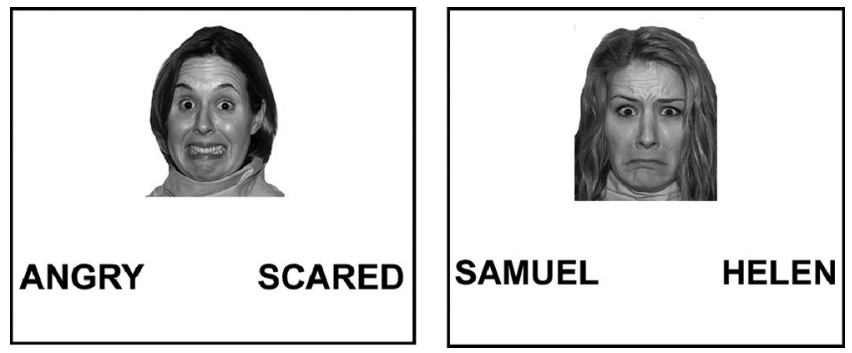

Figure 1. The left panel is an example of an affect labeling trial in which participants select the appropriate affect label characterizing the facial expression of the target face. The right panel is an example of a gender labeling (control) trial in which participants select the gender-appropriate name for the target face. well-being (e.g., empathy) and strong inverse associations with measures of physical symptoms and medical visit frequency (1). To examine the unique relationships between trait mindfulness and neural activity, the participants completed five additional measures that were used as control variables, given that mindfulness has been negatively associated with these measures in previous studies (1). These measures included: a) The Spielberger Trait Anxiety Inventory, a 20-item measure assessing long-term susceptibility to anxiety (sample $\alpha=0.91$ ) (17); b) a 10-item measure of neuroticism, drawn from the International Personality Item Pool (sample $\alpha=0.86$ ) (18); c) the Beck Depression Inventory, a 21-item measure assessing symptoms of depression over the past 2 weeks (sample $\alpha=0.88$ ) (19); d) the Global Severity Index of the Brief Symptom Inventory, a 52-item measure assessing feelings of distress over the last 2 weeks (sample $\alpha=94$ ) (20); and e) the Public Self-Consciousness subscale of the Self-Consciousness Scale, a 7-item measure assessing one's self-awareness as a social object (sample $\alpha=0.70$ ) (21).

\section{Data Acquisition and Analysis}

Neuroimaging data were acquired on a Siemens Allegra 3T head-only scanner. Head movements were restrained with foam padding and surgical tape across the forehead. For each participant, a high-resolution structural T2-weighted echo-planar imaging volume $($ spin-echo; $\mathrm{TR}=5000 \mathrm{~ms} ; \mathrm{TE}=$ $33 \mathrm{~ms}$; matrix size $128 \times 128$; 36 axial slices; FOV $=20$-cm; 3 -mm thick, skip 1-mm) was acquired coplanar with the functional scans. Two functional scans were acquired (echo planar $\mathrm{T} 2^{*}$-weighted gradient-echo, $\mathrm{TR}=3000$ $\mathrm{ms}, \mathrm{TE}=25 \mathrm{~ms}$, flip angle $=90^{\circ}$, matrix size $64 \times 64,36$ axial slices, FOV $=20-\mathrm{cm} ; 3-\mathrm{mm}$ thick, skip 1-mm). During each functional scan, 125 volumes were collected.

The imaging data were analyzed using Statistical Parametric Mapping (SPM'99, Wellcome Department of Cognitive Neurology, Institute of Neurology, London, UK). Images for each participant were realigned to correct for head motion, normalized into a standard stereotactic space as defined by the Montreal Neurological Institute (MNI), and smoothed with an 8-mm Gaussian kernel, full width at half maximum. For each participant, affect and gender labeling blocks were modeled as epochs. After the task was modeled for each participant, planned comparisons were computed as linear contrasts to investigate neural activity during the affect labeling, compared with the gender labeling condition. To create a mindfulness variable that controls for related individual differences measures, the control variables were regressed into the mindfulness variable and the standardized residuals were saved. These standardized residual values were then entered as a regressor in a random effects whole-brain group analysis, comparing neural activity during affect labeling with neural activity during gender labeling. Results are reported according to the voxel of peak activation among each identified cluster of activation. All analyses were carried out using an uncorrected $p=.005$ combined with a cluster size threshold of 10 voxels (22). All coordinates are reported in MNI coordinate space.

\section{RESULTS}

\section{Preliminary Analyses}

Correlational analyses examined associations between dispositional mindfulness and individual difference measures in the study survey battery. Dispositional mindfulness was negatively correlated with public self-consciousness $(r=-.51$; $p=.01$ ), but was not correlated with any of the other individual difference measures in the study (all $p>$.29). ${ }^{1}$ Further, dispositional mindfulness was not associated with ethnicity (when comparing Asians versus all other ethnic groups) $(t=$

\footnotetext{
${ }^{1}$ In examining the associations between mindfulness and sample demographic characteristics, we found a marginal association between gender and mindfulness $(r=.37 ; p=.06)$, with males reporting greater trait mindfulness. We do not believe that this effect represents a true population association, given that previous studies with large samples have found no such association (1). Nonetheless, we tested for significant gender $\times$ mindfulness interactions in all reported analyses and found no significant interactive effects. To minimize any confounding effects, all study analyses controlled for gender.
} 
$0.73 ; p=.48){ }^{2}$ Previous studies have found significant negative associations between mindfulness and measures of depression, anxiety, psychological distress, and neuroticism (1), so these measures (and gender) were used as control variables in all reported analyses. The results did not change appreciably when these control variables were excluded in study analyses.

\section{Behavioral Analyses}

Dispositional mindfulness was not correlated with reaction times during the affect labeling task $(r=-.29 ; p=.16)$ or the gender labeling tasks $(r=.002$; not significant (NS)). However, a weak association was observed during the affect labeling task, suggesting that greater mindfulness may be associated with faster affect labeling responses. This association may indicate an enhanced recognition and deployment of linguistic processing of affect in mindful individuals. Given the small number of errors produced by the labeling tasks ( $<2 \%$ error rates have been observed in previous studies), error rates were not recorded.

\section{Neural Analyses}

In the basic affect labeling $>$ gender labeling contrast, which has been reported previously (9), we observed activation in the right $\operatorname{VLPFC}(52,24,-10 ; \mathrm{t}=3.37 ; p<.005 ; k=31)$ and deactivation in the left amygdala $(-24,0,-24 ; t=-3.39$; $p<.005 ; k=56$ ). In comparison with the localized PFC activation observed previously, the present analyses indicated that greater levels of trait mindfulness were significantly associated with greater activity throughout the PFC during affect labeling compared with gender labeling. As shown in Table 1 and Figure 2, dispositional mindfulness was positively associated with activation in areas of right VLPFC, left VLPFC, ventromedial prefrontal cortex (VMPFC), MPFC, right dorsolateral prefrontal cortex (DLPFC), and the left insula. We also observed that dispositional mindfulness was associated with bilateral amygdala deactivation during affect labeling compared with gender labeling (left amygdala: $-16,0,16 ; r=-.61 ; p=<.001$; $k=73$; right amygdala: $22,2,-22 ; r=-.60 ; p=<.001 ; k=$ 19) (Figure 3).

Based on previous negative associations found between PFC and amygdala activity during affect regulation tasks (e.g., $10,11,13)$, connectivity analyses were conducted to test for relationships between the PFC and the amygdala as a function of mindfulness. To conduct these connectivity analyses, participants low $(n=14)$ and high $(n=13)$ in mindfulness were compared using a median split. Correlation analyses were conducted separately for the two groups between each identified PFC region (Table 1) and the amygdala to test for potential negative associations between these two regions. Analyses of the areas of activation associated with mindfulness demonstrated strong negative associations between several PFC regions and the

\footnotetext{
${ }^{2}$ We also tested for ethnicity (Asians versus non-Asians) $\times$ mindfulness interactions in all reported analyses and found no significant interactive effects.
}

TABLE 1. Neural Areas Associated With Mindfulness During Affect Labeling Compared to Gender Labeling

\begin{tabular}{lrrrrr}
\hline $\begin{array}{c}\text { Prefrontal Cortex } \\
\text { Region }\end{array}$ & \multicolumn{3}{c}{ MNI Coordinates } & Voxels & $\begin{array}{c}r \\
\text { Statistic }^{a}\end{array}$ \\
\hline MPFC (BA 9) & 2 & 60 & 28 & 2143 & .85 \\
Right VLPFC (BA 47) & 38 & 44 & 0 & 13 & .53 \\
Left VLPFC (BA 47) & -40 & 34 & -8 & 164 & .61 \\
VMPFC (BA 10) & 14 & 52 & -2 & 276 & .64 \\
Right DLPFC (BA 9) & 36 & 24 & 28 & 91 & .61 \\
Left insula & -26 & 16 & 18 & 183 & .74 \\
Left amygdala & -16 & 0 & 16 & 73 & -.61 \\
Right amygdala & 22 & 2 & -22 & 19 & -.60 \\
\hline
\end{tabular}

MNI = Montreal Neurological Institute; BA = Brodmann's area; MPFC = medial prefrontal cortex; VLPFC $=$ ventrolateral prefrontal cortex; $\mathrm{VMPFC}=$ ventromedial prefrontal cortex; $\mathrm{DLPFC}=$ dorsolateral prefrontal cortex.

${ }^{a}$ All correlations $(r)$ are significant at $p<.005$.

amygdala among participants high in mindfulness, whereas no such associations were found in participants low in mindfulness, suggesting that mindfulness may be associated with more efficient PFC inhibition of amygdala responses during affect labeling. Specifically, right VLPFC $(38,44,0)$ was negatively associated with right amygdala $(18,-2,-28)$ activity in participants high in mindfulness $(r=-0.88 ; p<.001)$, but not among participants low in mindfulness $(r=.20$, NS). Moreover, these correlational patterns were significantly different from each other (comparison of effects: $Z=3.61 ; p=$ .0003). Similar patterns of association between participants high and low in mindfulness were found for VMPFC and the right amygdala (VMPFC: $14,52,-2$; right amygdala: $14,-8$, -28 ; high mindfulness: $r=-.85 ; p<.001$; low mindfulness: $r=-.30$; NS; $Z=2.17 ; p=.03$ ), for MPFC and the right amygdala (MPFC: 2, 60, 28; amygdala: $14,-8,-28$; high mindfulness: $r=-.72 ; p=.005$; low mindfulness: $r=-.10$; NS; $Z=1.85 ; p=.06$ ), and right DLPFC and the right amygdala (DLPFC: 36, 24, 28; amygdala: 18, -2, 28; high mindfulness: $r=-.84 ; p<.001$; low mindfulness: $r=.25$; $\mathrm{NS} ; Z=3.38 ; p=.0007)$.

\section{DISCUSSION}

The present study provides the first neural evidence for associations among mindfulness, affect labeling, and improved neural affect regulation. Specifically, the present findings showed that dispositional mindfulness was associated with greater widespread PFC activation and attenuated amygdala responses during affect labeling. We also observed that participants high in mindfulness had strong inverse relationships between activity in these PFC regions and the right amygdala, whereas participants low in mindfulness did not show these effects. These findings build on previous emotion regulation findings (e.g., 13,14,16) and suggest that mindfulness may be associated with enhancements in these neural affect regulation pathways. Further, these findings are consistent with historical accounts of mindfulness, which suggest that affect labeling practices encourage individuals to treat 

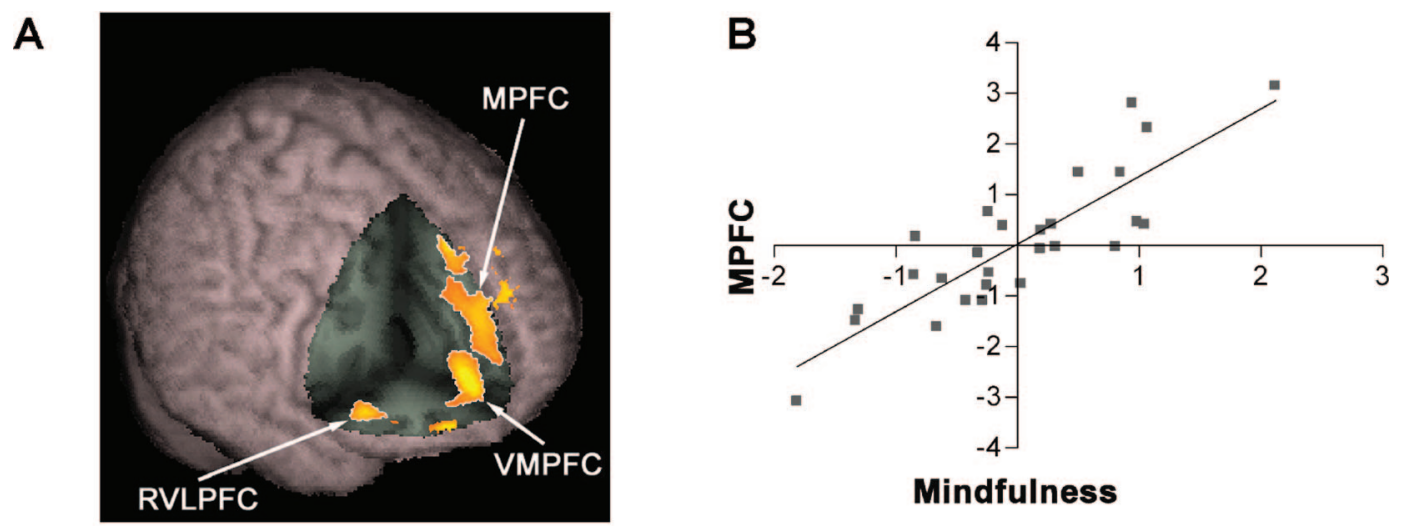

Mindfulness
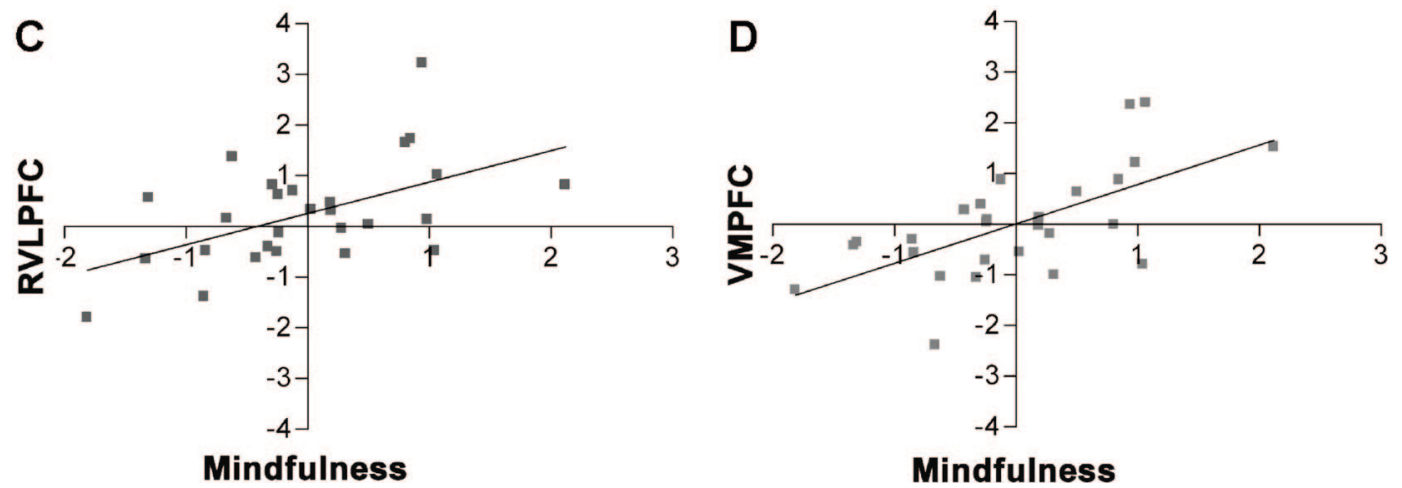

Figure 2. To illustrate the neural activations positively associated with mindfulness during the affect labeling $>$ gender labeling contrast, we highlight the effects found in the MPFC, right VLPFC, and VMPFC. In the upper left panel (A), arrows indicate activation in MPFC, right VLPFC (RVLPFC), and VMPFC. Surrounding this pictorial representation, scatterplots indicate neural activation in (B) MPFC, (C) right VLPFC, and (D) VMPFC, as a function of mindfulness. Each point represents the parameter estimates for a single participant's activity in the specified PFC region. MPFC $=$ medial prefrontal cortex; VLPFC $=$ ventrolateral prefrontal cortex; VMPFC = ventromedial prefrontal cortex.

affective states as "objects" of attention, thus promoting a certain amount of detachment from these negative states $(4,23)$. The most robust finding in the present study indicated a strong positive association between dispositional mindfulness and activation of the MPFC, a neural area that has been found to be activated during self-relevant tasks, such as monitoring one's own emotional state (24).

The present findings make an important contribution to the existing mindfulness literature by suggesting one neurocognitive pathway that may link mindfulness meditation practices with reductions in negative affect, mood disturbance, and physical symptoms across a number of patient populations $(3,24)$. One intriguing implication suggested by this work is that engaging in affect labeling during mindfulness meditation may improve prefrontal cortical regulation of limbic responses across a wide variety of situations encountered in daily life. Recent studies with experienced mindfulness meditation practitioners provide some preliminary support for this possibility $(25,26)$.

An important question that remains is how the present findings extend to the physical health domain. Recent studies in the behavioral medicine literature have shown that comparable neural activations to those found in the present study predict reductions in subjective reports of pain and anxiety and predict improvements in physical health outcomes (13-
16). For example, Urry and colleagues recently showed that cognitive reappraisal of negative pictures produced activation of MPFC and deactivation of the amygdala, and that this pattern of activation was associated with more adaptive diurnal cortisol patterns in a sample of older adults (13). Similarly, in a study on patients with chronic pain, increases in right VLPFC and corresponding decreases in limbic responses were associated with pain symptom improvements after a placebo treatment (15). In light of these findings, the data underscore the need for further research on the neurocognitive mechanisms of mindfulness-based treatments (as well as other behavioral treatments, e.g., psychotherapy, cognitive behavioral therapy) and their associations with physical health outcomes in targeted patient populations.

Some concerns can be raised about the conceptual validity and nature of our self-report measure of dispositional mindfulness and how it may relate to mindfulness meditation practice. Our present mindfulness measure captures a general disposition to be aware and receptive to present moment experiences, and increases with greater amounts of mindfulness meditation practice in meditation practice communities (1), and after mindfulness meditation interventions (27). Importantly, the present study used a residualized dispositional mindfulness measure which controlled for a number of individual difference measures, suggesting that unique variance 

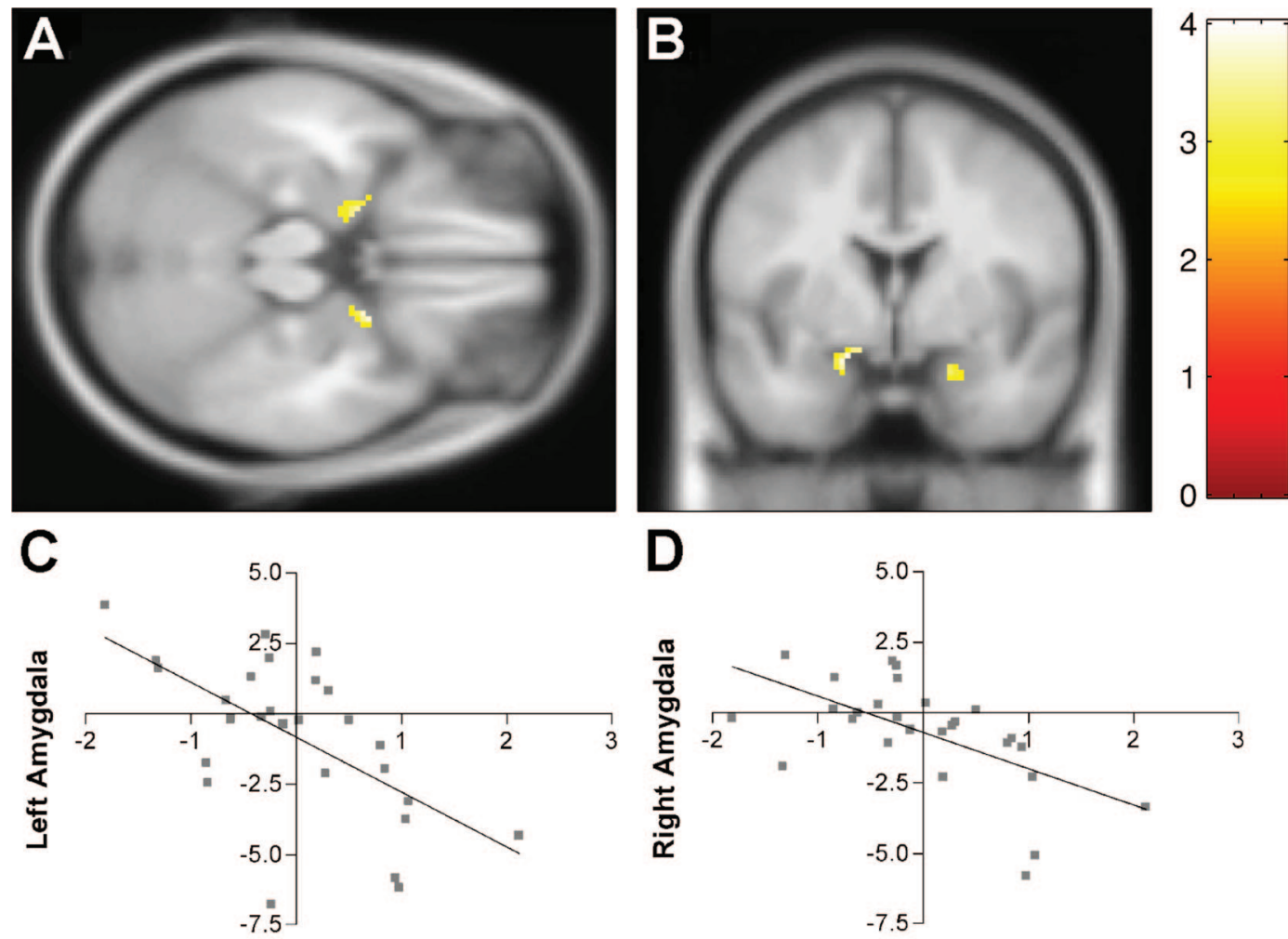

Mindfulness
D

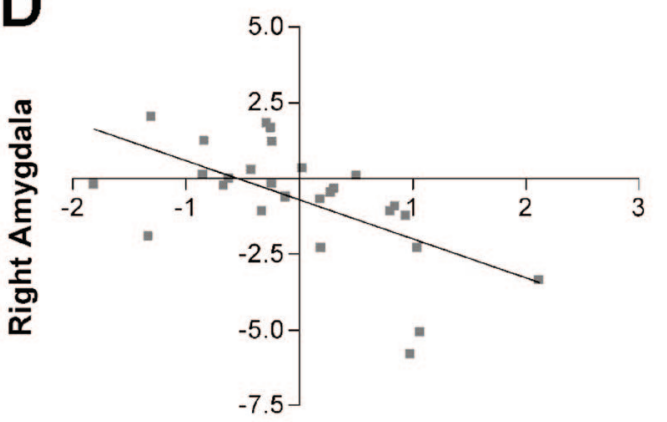

Mindfulness

Figure 3. In the top panel, bilateral amygdala activation that was negatively associated with mindfulness in the affect labeling $>$ gender labeling contrast viewed in an axial (A) and coronal (B) slice. In the bottom panel, scatterplots show the relationship between mindfulness and activity in the left (C) and right (D) amygdala. Each point represents the parameter estimates for a single participant's activity in the left amygdala $(-16,0,16)$ and right amygdala $(22,0,-22)$.

specific to dispositional mindfulness accounts for the present findings. A strong implication of these findings, to be tested in future research, is that dispositional mindfulness and mindfulness-enhancing interventions can reduce negative affect through affect labeling practices.

\section{CONCLUSIONS}

The present findings are part of the first efforts in understanding the neurocognitive underpinnings of mindfulness and in identifying the neural pathways that link mindfulness with improved psychological and physical well-being. These findings connect historical accounts of the Buddha's first teachings on mindfulness over two millennia ago (4) with contemporary findings in affective and cognitive neuroscience $(10,15)$, suggesting that mindfulness may reduce negative affect and promote greater physical health, in part, through labeling one's feelings.

The authors thank the UCLA Brain Mapping Center.

\section{REFERENCES}

1. Brown KW, Ryan RM. The benefits of being present: mindfulness and its role in psychological well-being. J Pers Soc Psychol 2003;84:822-48.

2. Nyanaponika. The heart of Buddhist meditation. New York: Weiser; 1962.

3. Baer RA. Mindfulness training as a clinical intervention: a conceptual and empirical review. Clin Psychol Sci Prac 2003;10:125-43.

4. Analayo. Satipatthana: the direct path to realization. Birmingham, UK: Windhorse Publications; 2003.
5. Goldstein J. Insight meditation: the practice of freedom. Boston, MA: Shambhala Press; 1993.

6. Segal ZV, Williams JMG, Teasdale JD. Mindfulness-based cognitive therapy for depression. New York: Guilford Press; 2002.

7. Linehan MM. Cognitive behavioral therapy of borderline personality disorder. New York: Guilford Press; 1993.

8. Baer RA, Smith GT, Allen KB. Assessment of mindfulness by selfreport: the Kentucky inventory of mindfulness skills. Assessment 2004; 11:191-206.

9. Lieberman MD, Eisenberger NI, Crockett MJ, Tom SM, Pfeifer JH, Way BM. Putting feelings into words: affect labeling disrupts amygdala activity to affective stimuli. Psychol Sci 2007;18:421-8.

10. Lieberman MD, Hariri A, Jarcho JM, Eisenberger NI, Bookheimer SY. An fMRI investigation of race-related amygdala activity in African-American and Caucasian-American individuals. Nat Neurosci 2005;8:720-2.

11. Hariri AR, Bookheimer SY, Mazziotta JC. Modulating emotional response: effects of a neocortical network on the limbic system. Neuroreport 2000;11:43-8.

12. Aron AA, Robbins TW, Poldrack RA. Inhibition and the right inferior frontal cortex. Trends Cogn Sci 2004;8:170-7.

13. Urry HL, van Reekum CM, Johnstone T, Kalin NH, Thurow ME, Schafer HS, Jackson CA, Frye CJ, Greischar LL, Alexander AL, Davidson RJ. Amygdala and ventromedial prefrontal cortex are inversely coupled during regulation of negative affect and predict diurnal pattern of cortisol secretion among older adults. J Neurosci 2006;26:4415-25.

14. Kalisch R, Wiech K, Critchley HD, Seymour B, O’Doherty JP, Oakley DA, Allen P, Dolan RJ. Anxiety reduction through detachment: subjective, physiological, and neural effects. J Cogn Neurosci 2005;17: $874-83$.

15. Lieberman MD, Jarcho JM, Berman S, Naliboff BD, Suyenobu BY, Mandelkern M, Mayer EA. The neural correlates of placebo effects: a disruption account. Neuroimage 2004;22:447-55. 
16. Ochsner KN, Bunge SA, Gross JJ, Gabrieli JDE. Rethinking feelings: an fMRI study of the cognitive regulation of emotion. J Cogn Neurosci 2002;14:1215-29.

17. Speilberger CD. Manual for the state-trait anxiety inventory (STAI). Palo Alto, CA: Consulting Psychologists Press; 1983.

18. Goldberg LR, Johnson JA, Eber HW, Hogan R, Ashton MC, Cloninger $\mathrm{CR}$, Gough HC. The international personality item pool and the future of public-domain personality measures. J Res Pers 2006;40:84-96.

19. Beck AT, Ward CH, Mendelson M, Mock J, Erbaugh J. An inventory for measuring depression. Arch Gen Psychiatry 1961;4: $561-71$.

20. Derogatis LR, Melisaratos N. The brief symptom inventory: an introductory report. Psychol Med 1983;13:595-605.

21. Fenigstein A, Scheier MF, Buss AH. Public and private self-consciousness: assessment and theory. J Con Clin Psychol 1975;43:522-7.

22. Forman SD, Cohen JD, Fitzgerald M, Eddy WF, Mintun MA, Noll DC. Improved assessment of significant activation in functional magnetic resonance imaging (fMRI): use of cluster-size threshold. Magn Reson Med 1995;33:636-47.

23. Titchener EB. Lectures on the elementary psychology of feeling and attention. New York: MacMillan; 1908.

24. Cahn BR, Polich J. Meditation states and traits: EEG, ERP, and neuroimaging studies. Psychol Bull 2006;132:180-211.

25. Lazar SW, Kerr C, Wasserman R, Gray JR, Greve D, Treadway M, McGarvey M, Quinn BT, Dusek JA, Benson H, Rauch SL, Moore CI, Fischl B. Meditation experience is associated with increased cortical thickness. Neuroreport 2005;16:1893-7.

26. Davidson RJ, Kabat-Zinn J, Schumacher J, Rosenkranz M, Muller D, Santorelli SF, Urbanowski F, Harrington A, Bonus K, Sheridan JF. Alterations in brain and immune function produced by mindfulness meditation. Psychosom Med 2003;65:564-70.

27. Cohen-Katz J, Wiley SD, Capuano T, Baker DM, Shapiro SL. The effects of mindfulness-based stress reduction on nurse stress and burnout, part II. Hol Nurse Pract 2005;19:26-35. 